\title{
Los equipos de orientación escolar en el nivel medio. Análisis de su funcionamiento en una escuela secundaria de gestión pública y otra de gestión privada de la provincia de Buenos Aires $^{1}$
}

\section{The school guidance teams at middle-school level. Analysis of their operation in a public management secondary school and in a private school in the Province of Buenos Aires}

Damián Pezzenati²

\begin{abstract}
Resumen: La escuela secundaria está estructurada en función de varios espacios organizacionales. Ya que el Equipo de Orientación Escolar (EOE) es uno de esos espacios, debe articular sus funciones con los demás estamentos de la estructura escolar. ¿Qué ocurre con la aparición del EOE en las instituciones educativas? ¿En qué medida cumple con las funciones explicitadas en le normativa oficial? ¿Cómo desarrolla sus actividades en una institución de gestión privada y en otra de gestión estatal? Este trabajo intenta dar respuesta a estos interrogantes, mediante el estudio y el análisis en dos instituciones educativas (una estatal y otra privada), acerca de cómo perciben los profesionales de ambas organizaciones el funcionamiento del EOE. A través de un
\end{abstract}

\footnotetext{
${ }^{1}$ Este artículo se basa en la Tesis de Maestría presentada por el autor en la Universidad Di Tella (ver Pezzenati, 2019).

2 Magíster y Especialista en Administración de la Educación. Licenciado y Profesor en Educación Física. Técnico Superior en Periodismo Deportivo. Vicedirector del Nivel Medio. Tigre, Buenos Aires, Argentina. Correo electrónico: damianpezzenati@gmail.com.
}

Diälogas Pedagágicas - ISSN en línea: 2524-9274.

Año XIX, No 37, abril-septiembre 2021. Pág. 53-75. DOI: http://dx.doi.org/10.22529/dp.2021.19(37)05 Recibido: 31-05-2020 / Aprobado: 3-11-2020.

(c) (i) $(-)$ Artículo publicado bajo Licencia Creative Commons Atribución-NoComercial-SinDerivar. cc) Universidad Católica de Córdoba. 
diseño metodológico de tipo descriptivo y de naturaleza cualitativa, donde el instrumento prioritario será la entrevista semiestructurada, con un carácter interpretativo y comparativo, este estudio pretende responder los interrogantes planteados.

Palabras clave: sistema educativo, distribución del espacio, estructura administrativa, gestión de personal.

Summary: High school is structured around various organizational spaces. Since the School Guidance Team (SGT) is one of those spaces, it must articulate its functions with the other levels of the school structure. What happens with the appearance of SGT in educational institutions? To what extent does it comply with the functions specified in the official regulations? How does it carry out its activities in a privately-managed institution and in another state-managed institution? This work tries to answer these questions, through the study and the analysis in two educational institutions, a state and a private institutions, about how the professionals from both organizations perceive the operation of the SGT. Through a methodological design of a descriptive and qualitative nature, where the priority instrument will be the semi-structured interview, with an interpretive and comparative nature, this study aims to answer the questions raised.

Keywords: education system, space arrangement, administrative structure, personnel management.

\section{INTRODUCCIÓN}

\section{Presentación del problema}

Según su tipo de gestión -estatal o privada- y los matices en cuanto a las características propias de cada institución escolar, la escuela secundaria en la provincia de Buenos Aires está, generalmente, estructurada en función de varios espacios organizacionales: la Dirección y la Vicedirección, la Dirección Pedagógica o Académica, la Secretaría, el Departamento de Alumnos, las coordinaciones de áreas o modalidades, los orientadores o tutores y el Equipo de Orientación Escolar (EOE). El nivel medio presenta la problemática de que cada espacio organizacional de su estructura funciona de manera aislada, siguiendo una lógica segmentada (Vicente, 2016). Cada docente con su materia, la Dirección y la Secretaría dedicadas a lo administrativo y los preceptores a la disciplina son un ejemplo de lo anterior. Trabajar hoy en la escuela secundaria implica abordar nuevas, permanentes y complejas situaciones para lo cual es necesario el aporte y el consenso de todos los actores educativos. Ya que el Equipo de Orientación Escolar es uno de los actores, debe articular sus funciones con los demás estamentos de la estructura escolar.

En el sistema educativo de la provincia de Buenos Aires, el Equipo de Orientación Escolar (EOE) tiene su origen en 2007. Surge como un nuevo dispositivo escolar que reemplazó a los llamados gabinetes psicopedagógicos. Desde la mirada estatal, estos fueron, por mucho tiempo, compartimentos aislados de la vida escolar. El propósito de profesores y directores era depositar allí las expectativas de modificaciones en el 
rendimiento académico o conductual de los educandos (Argentina, Ministerio de Educación, 2009). En las instituciones educativas, el EOE cumple su desempeño en todos los niveles de la enseñanza: inicial, primario y secundario. Su misión es la de intervenir en la atención de problemáticas concernientes a lo social, institucional y pedagógico-didáctico que involucren a los alumnos de todos los niveles y modalidades (Buenos Aires, Dirección General de Cultura y Educación, 2007). A su vez, presenta un cúmulo de saberes que provienen de diversos campos disciplinarios como la psicopedagogía, la psicología y el trabajo social, lo que marca cierta complejidad a la hora de articular sus funciones organizacionales con el resto de los espacios institucionales.

El propósito de este trabajo es observar y analizar lo que ocurre en las instituciones escolares con el funcionamiento de los EOE. Es interesante observar las dificultades y complejidades que acontecen en la práctica diaria, ya que, coincidiendo con Sileoni (2014), es necesario que se conforme una mirada institucional para repensar la demanda que le hace la escuela a los Equipos de Orientación Escolar y, también, para poder redefinir su objeto de trabajo. Mediante la descripción de sus roles y funciones, de su estructura organizativa y de la integración y articulación institucional, es como este trabajo, producto de una tesis de maestría, estudiará el funcionamiento del EOE en el nivel secundario. Para ello, se comparará lo que acontece en un colegio de gestión estatal y otro en el ámbito privado con el fin de echar luz sobre esta problemática.

\section{Antecedentes}

En este apartado, están descritos estudios relevantes que hacen referencia al tema de la investigación. Cabe aclarar que, si bien hay diversos estudios que analizan los Equipos de Orientación Escolar, esta sección se estructuró siguiendo un desarrollo que va desde los estudios realizados en nuestro país por Bayeto (2016), Greco (2014 y 2015), Bertoglio, Corizzo y Steiman (2017) a los trabajos de Sallán (1985) y Vélaz de Medrano Ureta et al. (2013), llevados a cabo en España. Estas publicaciones resaltan las problemáticas y desafíos de los Equipos de Orientación Escolar desde su creación hasta la incorporación oficial en las instituciones educativas.

Bayeto (2016) analiza el caso de los profesionales del Equipo de Orientación Escolar (EOE) en las escuelas estatales de la ciudad de Buenos Aires. En ese estudio, ofrece algunas claves para entender las modificaciones que se generaron, a través del tiempo, en las prácticas profesionales de sus miembros en el ámbito educativo. Describe algunas problemáticas que surgen a la hora de las intervenciones profesionales, como la "burocratización", el "automatismo de las derivaciones" y la "psicopatologización de los problemas educativos". El autor hace mención de que, si bien hay "un interés para la generación de propuestas y articulaciones mayores de las prácticas en las experiencias de las distintas instituciones, continúa prevaleciendo una considerable fragmentación en cuanto a las modalidades de intervención" (p. 18). A una conclusión similar llega Greco (2015) cuando menciona que las modalidades de intervención de los Equipos de Orientación Escolar en la provincia de Buenos Aires son dispares, dado que sus "equipos son numerosos, algunos insertos en las escuelas y otros, actuando a nivel distrital o supervisivo" (p. 156). Destaca, a su vez, que los EOE de la provincia de Buenos Aires son los más antiguos del país y los que poseen una mayor inserción en el ámbito educativo provincial. En este trabajo, compara las intervenciones del EOE en dos provincias argentinas: Buenos Aires y Río Negro, con distintas características geográficas, sociales y económicas, donde la conformación de los equipos es diferente; 
señala que, sin embargo, coinciden en exponer la misma problemática: ante el pedido de intervención, esta se ve "obstaculizada o interrumpida por la urgencia" (p. 158). La reformulación, por parte de los equipos de orientación, de sus marcos teóricos e instrumentales para generar cambios en sus propuestas que desemboquen en formas distintas de intervención es la alternativa viable para hacer frente a esta problemática (Greco, 2015).

En otro estudio, Greco (2014) explicita que hay muchas experiencias de los Equipos de Orientación Escolar en donde ensayan y experimentan modalidades de intervención institucional que implican revisar la manera de pensar la escuela, el alumno, el sistema educativo y la propia formación profesional. Destaca que, si bien puede ser considerado un avance, permanecen imperceptibles debido a la "dispersión de las acciones, escasa articulación con otros actores del sistema educativo, puesta en marcha de procesos y efectos institucionales que no son rápidamente constatables, etc." (p.154). Resalta que prevalece en el sistema educativo, en general, una modalidad de intervención de los Equipos de Orientación Escolar que da respuesta a demandas individuales de los problemas, enfocada desde una perspectiva reduccionista de la situación escolar. Abundando, "los equipos de orientación han mostrado como tendencia ofrecer respuestas predominantemente individuales a problemáticas que no dejan de ser institucionales, aunque emerjan en los sujetos o en entre ellos/as" (p. 153).

Bertoglio, Corizzo y Steiman (2017) explican que el cambio acontecido en la provincia de Buenos Aires, desde la denominación de "gabinete", en el pasado, hasta lo que hoy se define como Equipos de Orientación Escolar, generó una evolución en los modos de intervención: de una modalidad centrada en el "sujeto-problema" a trabajar en conjunto e interdisciplinariamente (p. 18). Señalan que se debe "resignificar la demanda" y que poder escuchar y "saber reconocer la demanda del otro y poder desde allí trabajar a fin de lograr proyectos comunes que atraviesen la institución propicia un mejor trabajo en equipo y mejor calidad para nuestros alumnos" (p. 19). Destacan que sería imposible para un equipo de orientación, que busca desempeñarse de manera colectiva y alejada del posicionamiento centrado en el "individuo-problema", no trabajar en comunión con docentes, preceptores, directivos y alumnos.

Es interesante el aporte que hace Sallán (1985) al investigar sobre el Departamento de Orientación, desde la perspectiva organizacional, en España. Explica que la tarea de orientar pasa, cada vez más, de una acción o función unipersonal a una acción de equipo. Señala que el Departamento de Orientación se piensa como el espacio que facilita la coordinación del conjunto de profesionales que intervienen en la tarea de orientar, lo que genera una acción cooperativa de los profesores. El autor menciona las ventajas del funcionamiento de este departamento: "posibilita la coordinación de actuaciones. Favorece la actualización y perfeccionamiento del profesorado. Permite una mayor rentabilidad de los medios. Integra y cohesiona al profesorado. Potencia la investigación operativa y mejora el conocimiento del objeto de estudio" (p. 39), aunque agrega que no es posible soslayar los problemas con respecto a su funcionamiento; esos son la "falta de falta de tradición y obligatoriedad legal. Escasez de tiempo disponible por el profesorado. El sentido individualista de las actuaciones docentes. La carencia de una infraestructura mínima. Las diferencias ideológicas entre el profesorado" (p. 39). Es útil la mirada que aporta el autor cuando refiere que, ante las diversas perspectivas que se tengan del proceso de orientación, la modalidad que se establezca en un establecimiento educativo es "en muchos casos, el resultado de un proceso de elección" (p. 50). 
Por último, Vélaz de Medrano Ureta et al. (2013) comparan la relación que existe entre la formación profesional de los miembros de los equipos de orientación y lo que demanda la tarea específica en los centros de educación primaria y secundaria. El estudio, que basó su análisis en las comunidades de Andalucía, Canarias, Cantabria, Castilla-La Mancha, Cataluña, Galicia, Madrid, Navarra y País Vasco, señala que una de las competencias profesionales que demanda el trabajo del orientador es el "trabajo en equipo-coordinación de equipos". Esto incluye la "coordinación de tutores y equipos directivos, dinámica de grupo y desarrollar estrategias de trabajo cooperativo" (p. 274).

Estos estudios citados muestran, por un lado, las mutaciones que han sufrido las funciones y prácticas de los profesionales del EOE en la institución educativa y, por otro lado, la prevalencia de una modalidad de intervención fraccionada y de tipo individual con los alumnos, lo que refuerza el concepto alumno-problema, que demanda la atención y su tratamiento. Esto dos aspectos atraviesan, en general, las prácticas del EOE en la escuela secundaria, más allá de que hay algunos casos aislados de instituciones educativas donde el EOE funciona de modo articulado con el resto de los actores educativos y realiza intervenciones institucionales.

\section{Justificación del problema}

Desde la sanción de la Ley Provincial № 13688 en 2007, que establece en el capítulo XII, título II, la conformación de Equipos de Orientación Escolar en todos los niveles y modalidades del sistema educativo provincial, no hay demasiados estudios que describan su gestión. Es importante observar, a más de una década de la sanción de la ley, cómo convive y actúa este equipo en el quehacer diario de la escuela secundaria.

El objetivo de los Equipos de Orientación Escolar lo señala la Ley 13688:

Conformar Equipos de Orientación Escolar en todos los establecimientos educativos a los efectos de intervenir en la atención de problemáticas sociales, institucionales y pedagógico-didácticas que involucren alumnos y grupos de alumnos de todos los Niveles y Modalidades del Sistema Educativo Provincial. (cap. 12)

Este objetivo está en sintonía con lo que demanda el trabajo de las escuelas hoy en día, para lo cual se requerirá del aporte concertado por todos los actores institucionales; entre ellos, el Equipo de Orientación Escolar colaborará con esta tarea.

No hace mucho tiempo, los Equipos de Orientación Escolar eran los llamados gabinetes. Eran entendidos como espacios o comportamientos aislados de la vida de la escuela, donde los directivos y los docentes depositaban las expectativas de cambio y transformación de las trayectorias escolares de los educandos. Pero, frente a la complejidad de la realidad que atraviesa hoy la escuela, estos espacios fueron creciendo y conformando distintos formatos que aparecen cada vez más como necesarios a la vez que más demandados (Ministerio de la Nación, Argentina, 2009).

Describir cómo funciona el Equipo de Orientación Escolar y cómo es su gestión contribuirá a comprender las problemáticas que ello conlleva. La incorporación de este nuevo órgano institucional, cuya función principal es la intervención con alumnos y grupos de alumnos en problemáticas sociales, institucionales y pedagógico-didácticas (Ley Provincial 13688), debe estar amalgamada con las estructuras escolares vigentes. Resulta muy pertinente analizar cómo se estructura la práctica y el campo de 
acción del EOE con los demás estamentos jerárquicos de la escuela: Dirección, Vicedirección, Secretaría, docentes y padres.

En los distintos estudios que existen sobre el tema, como los trabajos de Bayeto (2016), Bertoglio, Corrizo y Steiman (2017) y Greco (2014 y 2015), el enfoque se centra en las funciones de los miembros del EOE. Esto abarca tutorías, abordajes sobre distintas problemáticas de los adolescentes (adicciones y sexualidad), acompañamiento de las trayectorias escolares y atención de alumnos con problemáticas especiales (dislexia, déficit de atención, autismo, dislalia, etc.), relación con profesionales externos y alumnos integrados. Sin embargo, hay poco desarrollo en el campo práctico sobre cómo debe gestionarse y los alcances de los roles de sus integrantes.

De esta manera, el presente trabajo intentará responder, desde el estudio de dos realidades organizacionales distintas como lo son el ámbito estatal y el privado, qué pasa en la práctica con el EOE y qué contraste existe entre lo que expresa la ley y lo que ocurre en la realidad.

\section{Surgimiento del EOE en la provincia de Buenos Aires}

Los EOE hacen su aparición en la escena educativa de la provincia de Buenos Aires en 2007. Fue un proceso que se fue gestando desde los años noventa, una década marcada por políticas de descentralización y adecuación económica que impactaron en el sistema educativo y generaron como consecuencia una crisis en la institución escolar. La función y mandato de la escuela, en palabras de Fernández (2002, p. 17): "educar al soberano, alfabetizar y normalizar la cultura", sufría una deslegitimación. Ante este contexto, las instituciones educativas, conjuntamente con el Estado, realizaron modificaciones y transformaciones tendientes a pensar y a reflexionar sobre los fines y metas, los contenidos y metodologías de la enseñanza, el perfil del alumno, el rol del docente y los directivos, las nuevas formas de gestionar la escuela, el rol de los estudiantes y la problemática de la autoridad, entre otras cuestiones.

La respuesta a esa etapa de turbulencias en el ámbito educativo, que se evidenció en la década de los noventa con la Ley Federal de Educación $N^{\circ} 24125$, fue la promulgación de la Ley Nacional de Educación No 26206, en 2006, la Ley Provincial de Educación No 13688, en 2006, en 2011, la modificación del Reglamento General de las Instituciones Educativas de la provincia de Buenos Aires y, en los años subsiguientes, las alteraciones y cambios de los diseños curriculares del nivel secundario.

Los Equipos de Orientación Escolar surgen como resultado de estas transformaciones en el ámbito educativo. Anteriormente, existían los gabinetes psicopedagógicos que, basándose en un posicionamiento médico, atendían -de manera individual- problemáticas de conducta y aprendizaje. Castorina (2005) señala que el fundamento de esas prácticas era un conocimiento psicológico basado en el uso de una metodología individualista, que implicaba una visión escindida del sujeto. Como consecuencia de esto, se fue generando un abordaje de tipo clínico individual, sin atender las consideraciones y la participación de quienes intervenían en la enseñanza de los alumnos. De este modo, se asociaba y focalizaba la intervención en aquellos sujetos con dificultades. Una de las críticas a este modelo es la que aporta Baquero (2000) cuando lo describe como "la falacia de abstracción de la situación", que consiste en aislar las características de la situación educativa, escolar de los sujetos. Abundando, agrega lo siguiente: 
Como si pudieran delimitarse las posibilidades de ser educado -esto es de aprender y desarrollarse- de un sujeto sobre la base exclusiva de sus supuestos atributos personales. Recíprocamente, las situaciones educativas son vistas de modo naturalizado o cosificado, como si consistieran realmente en contextos naturales de aprendizaje. (p. 11)

Bertoglio, Corizzo y Steiman (2017), en sintonía con lo anterior, comentan que las intervenciones consistían en aislar o excluir al alumno del grupo de clase para poder trabajar con él de manera individual, lo que conllevaba no tener en cuenta otros factores que inciden en el comportamiento-actitudes del sujeto y en el rendimiento académico. Señalan que, para superar esta posición, es menester que las acciones que se lleven a cabo sean en relación con otros, con los demás actores institucionales, lo que implica trabajar interdisciplinariamente.

En cambio, el EOE refiere a la noción de equipo, que según Claudia Bello:

Habla de un paradigma de promoción y protección de derechos de niños y adolescentes (...) Al pensar a la educación como un bien social, planificamos un trabajo de los Equipos que no parte desde lo individual, porque la subjetividad se construye con los otros en un proceso de intersubjetividad. Se pasa de una práctica de consultorio a una práctica más abierta, más comunitaria y de intervención grupal. (2010, p. 37)

En 2006, comienza a reglamentarse la conformación y las funciones del EOE. La Ley de Educación Nacional N²6206 -además de fijar la obligatoriedad de la escuela secundaria en todo el país- en su artículo 32, fija disposiciones para la atención psicopedagógica, psicológica y médica de adolescentes y jóvenes que lo necesiten por medio de gabinetes interdisciplinarios en los establecimientos educativos y la coordinación entre distintas áreas de gobierno de políticas sociales (Ley de Educación Nacional No 26206, p. 7). En 2007, la Ley Provincial No 13688, en su artículo 43, del capítulo XII: Psicología Comunitaria y Pedagogía Social, precisa que esta consiste en lo siguiente:

La modalidad con un abordaje especializado de operaciones comunitarias dentro del espacio escolar, fortalecedoras de los vínculos que humanizan la enseñanza y el aprendizaje; promueven y protegen el desarrollo de lo educativo como capacidad estructurante del sujeto y de la comunidad educativa en su conjunto, respetando la identidad de ambas dimensiones desde el principio de igualdad de oportunidades que articulen con la Educación común y que la complementen, enriqueciéndola. ( $p .16$ )

Dentro de este artículo, se especifican sus funciones y objetivos. Entre ellos, se explicita la creación de Equipos de Orientación Escolar en todos los establecimientos y niveles del sistema educativo de la provincia de Buenos Aires. Menciona que la intervención de los EOE será sobre problemáticas sociales, institucionales y pedagógico-didácticas de alumnos o grupos de alumnos.

La Disposición del 8 de septiembre de 2008, "Rol del Equipo de Orientación Escolar", precisa que los equipos estarán conformados por: orientador social, maestro/a recuperador/a u orientador de aprendizaje, fonoaudiólogo/a u orientador fonoaudiológico y médico/a u orientador médico. Destaca la disposición que las intervenciones deben tener un carácter interdisciplinar e interinstitucional y que las tareas que se lleven a cabo serán de manera conjunta. Esta modalidad de trabajo tiene como denominador 
común la articulación e interdisciplinariedad. Con relación a este último término, se señala que "no es la yuxtaposición ni una suma de saberes sino una puesta en común, una forma de conocimiento aplicado que se produce en la intersección de un saber con otro u otros" (p. 1).

La articulación será primordial y, junto con la interdisciplinariedad, modelan una forma especial de trabajar del EOE que implica la intervención profesional mediante la aplicación de conocimientos que derivan de la confluencia de distintos saberes. Por lo tanto, hay un trabajo en conjunto que es fundamental para la resolución de las distintas problemáticas en las que interviene el EOE. En el artículo 4, se menciona que una de las funciones del EOE es la de planificar, anualmente, un proyecto integrado de intervención en forma conjunta, "respetando la especificidad de cada rol, las características institucionales, las comunitarias y del Nivel Educativo y Modalidad en el que se inserta" (p. 2). Finalmente, el artículo 7 expone la participación de los miembros del EOE en distintas actividades de la vida institucional, como la elaboración del Proyecto Educativo Institucional (PEI) y de los Acuerdos Institucionales de Convivencia (AIC) y la generación de oportunidades para que los alumnos terminen sus estudios en los distintos niveles del sistema educativo. La organización y realización de reuniones, por un lado, entre el personal docente y directivo, para brindar herramientas y estrategias de intervención de acuerdo con las necesidades y expectativas de la comunidad escolar, para proponer y colaborar en proyectos pedagógicos y para analizar situaciones grupales e individuales acerca de las trayectorias escolares de los alumnos. Y, por otro lado, llevar a cabo reuniones de padres para ofrecer orientaciones y acompañamiento a los alumnos.

Por su parte, la Disposición No 1 de 2009 de la provincia de Buenos Aires especifica más las funciones del EOE. En los artículos 2 y 3, explica en qué centrarán su función en el nivel inicial y primario, respectivamente. En su artículo 4, señala que los Equipos de Orientación Escolar en la escuela secundaria se enfocarán en temas como la retención y promoción de los alumnos, la participación en la construcción de los Acuerdos Institucionales de Convivencia (AIC), la prevención de problemáticas infanto-juveniles y en la orientación para la educación y el trabajo (ofertas educativas y articulación con el mundo del trabajo). Finalmente, explica que "Los E.O.E. que se desempeñan en Primaria cooperarán con la Secundaria si ésta se encuentra en el mismo edificio y no posee por el momento E.O.E., definiendo sus motivos de intervención" (artículo 5, Disposición 1/09, p.3).

Por último, el Reglamento General de las Instituciones Educativas de 2012, en los artículos 82, 83 y 84, en el apartado Cargos técnico-docentes de base, menciona los diferentes profesionales que pueden formar parte del EOE y su ámbito de incumbencia. Menciona las tareas que llevarán a cabo, especificando que las prescripciones detalladas se aplicarán conforme a adecuaciones que correspondan a cada nivel y modalidad de desempeño (artículo 84, p. 42).

\section{Objetivo general de la tesis}

Analizar el funcionamiento del Equipo de Orientación Escolar como estructura que forma parte de la institución educativa, inserto en una organización que aprende, a través del estudio de dos escuelas secundarias de la provincia de Buenos Aires, una de gestión privada y otra estatal. 


\section{Objetivos específicos}

Detallar cuáles son las funciones del EOE en ambas instituciones y analizar en qué medida cumplen con lo dispuesto en la normativa oficial.

Analizar cómo se articula el funcionamiento del EOE de ambos colegios con los roles de dirección y supervisión, reconociendo el modelo de gestión institucional en donde está inserto.

Analizar la dinámica de funcionamiento interno del EOE en cada institución.

Corroborar en qué medida el trabajo del EOE de cada colegio se ajusta a los lineamientos de una organización escolar que aprende.

\section{MÉTODO}

Este trabajo implicó el estudio y el análisis de dos instituciones educativas, por lo que el diseño metodológico que se utilizó fue el de tipo descriptivo. Este tuvo una naturaleza cualitativa y el instrumento prioritario fue la entrevista semiestructurada, a la vez que contó con un carácter interpretativo y comparativo. Esto permitió realizar una adecuada recolección de los datos, lo que posibilitó alcanzar los objetivos propuestos y poder responder a la pregunta de investigación planteada.

La atención de esta investigación se centró en sistematizar y analizar las entrevistas realizadas a los miembros de los Equipos de Orientación Escolar, a profesores y a miembros del equipo directivo de ambas instituciones educativas. Las preguntas seleccionadas intentan producir información acerca del funcionamiento, la inserción y la articulación del EOE en la estructura de la escuela secundaria. En ese marco, surgieron, por un lado, algunas cuestiones primarias que guiaron este trabajo, tales como: ¿Cómo será la modalidad de trabajo del EOE en un colegio de gestión estatal y otro privado?, ¿qué relación tendrá con el equipo directivo?, ¿qué lugar ocupará en el organigrama institucional?, ¿la conformación de los EOE será la misma en ambas instituciones?, ¿qué grado de participación tiene el EOE en la vida institucional? Y, por otra parte, se construyeron variables de investigación que permitieron guiar y direccionar el análisis de este estudio. Estas fueron las siguientes: Grado de articulación con las distintas estructuras de la institución; conformación del EOE y sus roles; condiciones laborales y jerarquización del rol; funciones, actividades y tareas que lleva a cabo el EOE; grado de intervención de las funciones del EOE en la institución y percepción y pensamientos de los miembros acerca del funcionamiento del EOE. La tabla 1 muestra las variables y su explicación. 
Tabla 1. Variables de la investigación

\begin{tabular}{|c|c|}
\hline Variables & Definición \\
\hline $\begin{array}{l}\text { Grado de articulación con las distintas es- } \\
\text { tructuras de la institución }\end{array}$ & $\begin{array}{l}\text { El EOE en la organización del colegio. Des- } \\
\text { cribe como es la relación e interacción con } \\
\text { el equipo directivo y las demás coordinacio- } \\
\text { nes. }\end{array}$ \\
\hline Conformación del EOE y sus roles & $\begin{array}{l}\text { Evidencia y describe la composición del EOE } \\
\text { y qué actividades desempeñan. }\end{array}$ \\
\hline $\begin{array}{l}\text { Condiciones laborales y jerarquización del } \\
\text { rol }\end{array}$ & $\begin{array}{l}\text { Es el modo en que la actividad se desarro- } \\
\text { lla. Evidencia disponibilidad horaria y si es } \\
\text { trabajo en planta funcional o extraprogra- } \\
\text { mático. Estas condiciones están en estrecha } \\
\text { relación con la jerarquía organizacional de } \\
\text { la escuela. }\end{array}$ \\
\hline $\begin{array}{l}\text { Funciones, actividades y tareas que lleva a } \\
\text { cabo el EOE }\end{array}$ & $\begin{array}{l}\text { Describe las actividades que llevan a cabo } \\
\text { sus miembros en la institución educativa. } \\
\text { Están en concordancia con el desempeño de } \\
\text { rol. }\end{array}$ \\
\hline $\begin{array}{l}\text { Grado de intervención de las funciones del } \\
\text { EOE en la institución }\end{array}$ & $\begin{array}{l}\text { Explica cuándo participa el EOE en la de- } \\
\text { manda del colegio. Describe las situaciones } \\
\text { y motivos que generan la intervención del } \\
\text { EOE. }\end{array}$ \\
\hline $\begin{array}{l}\text { Percepción y pensamientos de los miembros } \\
\text { acerca del funcionamiento del EOE }\end{array}$ & $\begin{array}{l}\text { Describe cómo evalúan sus miembros al } \\
\text { EOE. Evidencia satisfacción o no en el desa- } \\
\text { rrollo de la tarea diaria. }\end{array}$ \\
\hline
\end{tabular}

Los casos analizados fueron dos colegios de distintos partidos de la zona norte del conurbano bonaerense; uno ubicado en San Isidro y el otro, en Tigre; el primero de gestión privada y el otro, estatal. La razón de la elección de estas instituciones se centró en la intención de corroborar cómo realizaban sus funciones los Equipos de Orientación Escolar en ámbitos tan distintos como lo son las instituciones educativas de gestión estatal y las de gestión privada. En nuestro sistema educativo, prima la heterogeneidad, evidenciada en factores culturales, económicos y sociales que ocasionan diferencias entre los distintos establecimientos escolares y entre el propio alumnado. Como resultado, los procesos de enseñanza-aprendizaje que transitan los educandos serán desiguales. Cervini (2002) menciona que lo anterior es consecuencia, por un lado, de las particularidades específicas de los niños-jóvenes y las condiciones socioeconómicas de origen que poseen y, por el otro, el efecto que la escuela ocasiona sobre las trayectorias escolares.

En la dualidad planteada, colegio público - colegio estatal, los EOE deben insertarse y desplegar sus funciones en realidades muy distintas en cuanto al tipo de gestión, los recursos y la estructura organizacional. Por eso, se elige, en esta investigación, un colegio privado del partido de San Isidro, sin subvención, porque interesa observar cómo se desempeña el EOE dentro de una institución cuya forma de organización y 
funcionamiento presenta márgenes destacados de flexibilidad para luego poder contrastar y comparar con un colegio público donde lo normativo, la estructura organizacional y su funcionamiento evidencian signos de rigidez burocrática. El análisis que se haga sobre el EOE dentro de esta dicotomía es relevante, pues permitirá echar luz sobre su funcionamiento y articulación organizacional en ambas modalidades de gestión.

Con respecto a la técnica de recolección de datos, se realizaron observaciones y se desarrollaron entrevistas con actores institucionales; también existió la posibilidad del análisis documental de entrevistas realizadas por el EOE. No se desechó la posibilidad de que pudieran haber surgido datos de otras fuentes primarias, como los obtenidos por la participación en reuniones que los miembros del EOE llevaron a cabo con el equipo directivo o con los profesores, o datos que surgieron por la intervención en alguna dinámica que el EOE llevó a cabo con alumnos. Las técnicas de análisis de los datos que se emplearon fueron el análisis de contenido y la codificación. El análisis de los datos que se obtuvo de las fuentes mencionadas anteriormente conllevó la sistematización de la información, la examinación, contrastación o comparación de los datos recogidos en ambas instituciones.

Para la identificación de los datos que se obtuvieron de las distintas entrevistas a docentes, miembros del equipo directivo y del EOE, se utilizó lo que proponen Maykut y Morehouse (1994): la comparación y agrupación de las respuestas a cada interrogante planteado, relacionadas a una misma pregunta, para luego hacer confluir las respuestas con cada una de las variables de la investigación, según el punto en común que manifiesten con el patrón establecido. También, se tomó en cuenta lo que menciona Ruiz del Cerro (2000) sobre las líneas de acción en una investigación cualitativa: distribuir, mediante el análisis reflexivo, los datos arrojados por los entrevistados y organizarlos de manera tal que se puedan identificar contenidos de relevancia para el investigador. Por lo tanto, el proceso siguió estos pasos: separación de datos, reconocimiento y clasificación de patrones-unidades y, por último, procesamiento y elaboración de los datos en un esquema que permita organizar y comprender esos datos e ideas de manera significativa.

Por último, con respecto a los recursos, se recurrió a contactos establecidos con los directores de ambas instituciones educativas para poder acceder al análisis del EOE en cada una ellas.

\section{Las entrevistas}

El objetivo de las entrevistas fue obtener información de los actores institucionales con respecto a lo siguiente: el grado de articulación con las distintas estructuras de la institución; la conformación del EOE, roles y funciones; las condiciones laborales y jerarquización del rol; las funciones-actividades y tareas que lleva a cabo el EOE; el grado de intervención de las funciones del EOE en la institución; la percepción y pensamientos de los miembros acerca del funcionamiento del EOE; las tareas y funciones que lleva a cabo el EOE. Para ello, el análisis se centró en el estudio de tres grupos: el equipo directivo, los profesores y el Equipo de Orientación Escolar. La elección de este tipo de entrevista se basó en la pretensión de que los entrevistados se explayaran en sus respuestas y apreciaciones. También, interesaba saber qué grado de familiaridad tenían con el tema de estudio, más allá de sus funciones y roles laborales específicos. 
Los entrevistados, en ambas instituciones, fueron un total de diez personas. En el colegio de gestión estatal, se interrogó a la directora, a la secretaria, a la psicopedagoga y a siete profesores; en el colegio de gestión privada, se entrevistó a la directora, a la vicedirectora, al coordinador del Departamento de alumnos, a la psicopedagoga, al psicólogo y a cinco profesores. La siguiente tabla muestra las preguntas para ser respondidas por los directivos, los miembros del Equipo de Orientación Escolar, los profesores y su relación con las variables de la investigación (Ver Tabla 2).

Tabla 2. Guía de preguntas y la relación con las variables de la investigación

\begin{tabular}{|c|c|}
\hline Preguntas & $\begin{array}{l}\text { Variables sobre las que se } \\
\text { intenta recabar información }\end{array}$ \\
\hline $\begin{array}{l}\text { ¿Con qué estructura organizacional se vincula el } \\
\text { Equipo de Orientación Escolar? ¿Cómo se desarrolla } \\
\text { la articulación con los diferentes órganos de gestión } \\
\text { institucional? ¿En qué lugar de la estructura jerárquica } \\
\text { organizacional está el EOE? ¿Qué relevancia tiene a ni- } \\
\text { vel organizacional? }\end{array}$ & $\begin{array}{l}\text { El grado de articulación con las } \\
\text { distintas estructuras de la insti- } \\
\text { tución }\end{array}$ \\
\hline $\begin{array}{l}\text { ¿Quiénes conforman el EOE? ¿Cuáles son los roles } \\
\text { que llevan a cabo? }\end{array}$ & Conformación del EOE y sus roles \\
\hline $\begin{array}{l}\text { ¿Cuáles son las condiciones laborales del EOE } \\
\text { (disponibilidad horaria de los integrantes, personal de } \\
\text { planta o extracurricular, asesoramiento externo (mono- } \\
\text { tributistas))? ¿Cómo fue la selección de los miembros } \\
\text { del EOE? }\end{array}$ & $\begin{array}{l}\text { Condiciones laborales y jerarqui- } \\
\text { zación del rol }\end{array}$ \\
\hline $\begin{array}{l}\text { ¿Cómo trabaja el Equipo de Orientación Escolar } \\
\text { (EOE) (procedimientos, tareas específicas, deriva- } \\
\text { ciones, etc.)? Sobre estos aspectos vinculados con la } \\
\text { gestión institucional: ¿Proyecto Educativo Institucional } \\
\text { - Coordinación pedagógica con profesores - Disciplina } \\
\text { escolar -Acuerdo de Convivencia Institucional- Trabajo } \\
\text { con padres y comunidad escolar - Evaluación de los } \\
\text { estudiantes? ¿En qué áreas o ámbitos trabaja general- } \\
\text { mente el EOE? ¿Con qué criterios son distribuidas las } \\
\text { tareas en el EOE? }\end{array}$ & $\begin{array}{l}\text { Sobre funciones-actividades y ta- } \\
\text { reas que lleva a cabo el EOE }\end{array}$ \\
\hline $\begin{array}{l}\text { ¿Cuáles son las intervenciones (o consultas) más } \\
\text { recurrentes que recibe el EOE? En las reuniones ins- } \\
\text { titucionales que participa o lleva a cabo el EOE, ¿cuáles } \\
\text { son los asuntos más frecuentemente tratados? ¿Cuáles } \\
\text { son los de mayor conflictividad? }\end{array}$ & $\begin{array}{l}\text { Grado de intervención de las fun- } \\
\text { ciones del EOE en la institución }\end{array}$ \\
\hline $\begin{array}{l}\text { ¿Cuál es su visión actual sobre el EOE en la institu- } \\
\text { ción? ¿Cuáles serían los factores fundamentales para } \\
\text { la gestión del EOE? ¿Qué balance hace sobre el funcio- } \\
\text { namiento del EOE? Desde que se instaló o creó el EOE } \\
\text { en la escuela, ¿qué cambios se han generado a partir } \\
\text { de su aparición en el nivel secundario? }\end{array}$ & $\begin{array}{l}\text { Percepción y pensamientos de } \\
\text { los miembros acerca del funcio- } \\
\text { namiento del EOE }\end{array}$ \\
\hline
\end{tabular}




\section{Características del colegio de gestión pública - Colegio A}

El colegio surge de una necesidad de la comunidad de Tigre, en la década de 1950, de contar con un establecimiento de educación secundaria. En ese tiempo, una comisión cooperadora conformada por padres de alumnos y vecinos activó la creación y la puesta en marcha. Estas gestiones generaron que, en 1959, el Ministerio de Educación de la provincia de Buenos Aires cediera al Municipio de Tigre un inmueble en la zona residencial. Luego, el partido de Tigre lo cedió al Ministerio de Educación de la Nación. Posteriormente, ante la visita del inspector distrital, el edificio obtuvo el apto para su funcionamiento.

La comisión cooperadora, con posterioridad, emprendió la búsqueda de subsidios para realizar las reparaciones y remodelaciones necesarias en la propiedad. A través de un subsidio municipal, en 1960, obtuvo los recursos económicos para la realización de las obras. Los padres de los futuros alumnos aportaron la mano de obra para los distintos trabajos de albañilería, pintura, plomería y otros y el Rotary Club de Tigre donó libros para la formación de la biblioteca. Durante este proceso de obras, comenzó la inscripción de los alumnos y el 21 de marzo de 1960 se aprueba el nombre del colegio (para este trabajo, Colegio B). El 4 de abril del mismo año, se inaugura oficialmente esta institución.

En un comienzo, el colegio contaba con cuatro divisiones distribuidas en el turno mañana y el turno tarde. Por la mañana, estaban 1er, 2 do y 3er años y, en el turno tarde, otra división de 1er año. Por ese entonces, tenía una matrícula de casi ochenta alumnos. Con el correr de los años y como consecuencia de un fuerte crecimiento de la matrícula, la institución completó el ciclo en ambos turnos. En 2018, la matrícula alcanzó a casi mil trescientos alumnos distribuidos en cuatro divisiones hasta completar la ESB (Educación Secundaria Básica) y en cuatro modalidades: Comunicación, Economía, Sociales y Naturales al término de la ESO (Educación Secundaria Orientada). En contraturno, tanto para la ESB como para la ESO, se desarrolla la materia de Educación Física en las instalaciones de un polideportivo municipal cercano al colegio. Además, tiene una oferta variada de talleres extraprogramáticos: escuela deportiva, ajedrez, computación, música, teatro, inglés y apoyo escolar.

La selección y la ocupación de los cargos, tanto para el equipo de conducción como para el plantel de profesores y el personal no docente, se realiza mediante concurso - acto público que establece el Consejo Escolar de Tigre según la disponibilidad y demanda del establecimiento educativo. El equipo de conducción está conformado por la directora, la vicedirectora, la secretaria y la prosecretaria y el jefe de preceptores que coordina a doce preceptores distribuidos uno cada dos cursos. El Equipo de Orientación Escolar está conformado por una psicóloga y una psicopedagoga. Para algunas situaciones que el colegio evalúa como serias y riesgosas (por ejemplo, abandono escolar, violencia familiar, abuso y adicción), ¿se notifica al inspector de distrito quien convoca a un asistente trabajador social para acompañar y asesorar al equipo de conducción en la problemática demandada?

El colegio cuenta con dos plantas; en el primer piso, está la educación secundaria básica y, en la planta alta, la educación secundaria orientada. En la planta baja, se encuentran las doce aulas de 1er a 3er año, más el taller de arte, el laboratorio de Informática, la biblioteca, el buffet y los baños para esa sección. No cuenta con comedor. Las aulas rodean el patio central, que cuenta con un mástil donde se lleva a cabo la 
formación de la mañana. En la planta alta, están las aulas para los cursos superiores, los sanitarios y una sala de video.

El equipo directivo comenta que, en estos últimos años, el ausentismo docente es alto. Hay un porcentaje alto de suplentes. Las suplencias, a veces, tardan en tomar posesión del cargo debido a demoras burocráticas. Esto lo remarcan como algo perjudicial en el desarrollo de los contenidos curriculares a trabajar con los alumnos.

\section{Características del colegio de gestión privada - Colegio B}

La institución pertenece al obispado de San Isidro y está ubicada cerca del casco histórico de dicha localidad. Brinda una propuesta educativa de jornada simple extendida, desde el Nivel Inicial hasta el Secundario. No percibe subvención estatal. Tiene un plan de bonificaciones en los aranceles si uno o ambos padres trabajan en la institución, también si son dos o más hermanos y por el pronto pago a través del débito automático. Brinda, también, un porcentaje de becas (aprobadas por la Dirección General) según el análisis de situaciones familiares que se realicen.

La formación religiosa es un pilar central en el proyecto educativo institucional. El alumnado vive la pastoral del colegio a través de celebraciones, retiros, jornadas espirituales, padrinazgo, misiones y otros espacios de formación cristiana. También lo remarca su misión institucional, en donde se explicita que es un colegio que pertenece a la Iglesia católica y que, inspirados en el Evangelio, educa a personas en comunidad para que sean competentes, compartan sus dones y talentos y puedan así transformar la realidad.

La mayoría de los padres de los alumnos cuenta con formación terciaria y universitaria. La participación de las familias en el colegio es muy alta, tanto en las actividades que propone la institución como las que plantean y demandan las familias. Existe una unión de padres representada por doce padres/madres (un padre/madre por cada curso) que mantiene reuniones mensuales con la Dirección General y los equipos directivos de cada nivel para plantear inquietudes y recibir respuestas institucionales. Es la vía de comunicación con el resto de las familias del colegio.

En la escuela secundaria, hay doce secciones, dos por nivel, y tiene una matrícula de doscientos cincuenta y siete alumnos. Cuenta con dos orientaciones en la educación secundaria orientada, la modalidad de Comunicación y la de Ciencias Sociales. Tiene una oferta extracurricular de talleres obligatorios de distintas áreas, donde los alumnos cursan en forma cuatrimestral y/o anual distintas materias para completar su formación. El listado de los talleres es el siguiente: Inglés, Informática, Lenguajes artísticos, Práctica contable, Gestión administrativa y Proyecto de investigación. La estructura del nivel está formada por la directora y la vicedirectora, el coordinador del Departamento de alumnos y seis preceptores del nivel, distribuidos uno por cada año, y el Departamento de Orientación Psicopedagógico (DOP). Este nombre, en lugar de Equipo de Orientación Escolar (EOE), fue establecido en 2010 y hoy día, en la práctica cotidiana institucional, se lo sigue denominando de este modo. Está formado por una psicopedagoga, un psicólogo y dos orientadores-tutores. Estos acompañan la trayectoria escolar de los alumnos desde el ingreso hasta el egreso del secundario.

El colegio cuenta con dos plantas; en el primer piso, está la Educación Primaria $y$, en la planta alta, el Secundario. Tiene un patio central en la planta baja, donde se desarrollan los recreos de ambos niveles. También, en ese sector, se encuentra la 
Secretaría, la oficina de la Dirección General, la oficina del DOP, el laboratorio de Informática, el salón de Arte y Música y el teatro. Hay un pequeño buffet que está junto al patio de recreo. En el primer piso, están las aulas, las oficinas de la Dirección y del coordinador del Departamento de alumnos, la sala de profesores y el despacho de los preceptores. Los docentes, en su mayoría, son titulares; hay un bajo porcentaje de solicitudes de licencia. Las suplencias son esporádicas. Hay poco ausentismo docente.

La directora comenta que el obispado de San Isidro se hizo cargo de la administración del colegio en 1979. Anteriormente, estaba bajo el control de los Hermanos de las Escuelas Cristianas, cuya competencia -en materia educativa- era notoria en el país, particularmente por el Colegio La Salle de la ciudad de Buenos Aires. Comenta que es una de las instituciones más antiguas y tradicionales de San Isidro.

\section{RESULTADOS}

\section{Colegio A}

Tabla 3. Datos recolectados en el Colegio A

\begin{tabular}{|c|c|c|c|}
\hline Variables & Equipo directivo & EOE & Profesores \\
\hline $\begin{array}{l}\text { El grado de ar- } \\
\text { ticulación y vin- } \\
\text { culación con las } \\
\text { distintas estruc- } \\
\text { turas de la insti- } \\
\text { tución }\end{array}$ & $\begin{array}{l}\text { Depende del Equi- } \\
\text { po directivo. Se lo } \\
\text { convoca para tratar } \\
\text { problemáticas gru- } \\
\text { pales e individuales } \\
\text { de los alumnos. }\end{array}$ & $\begin{array}{l}\text { Conocen dónde se ubi- } \\
\text { ca el EOE en la estruc- } \\
\text { tura organizacional. }\end{array}$ & $\begin{array}{l}\text { Desconocen. Algu- } \\
\text { nos mencionan que } \\
\text { es parte del staff de } \\
\text { la Vicedirección. }\end{array}$ \\
\hline $\begin{array}{l}\text { Conformación } \\
\text { del EOE y sus ro- } \\
\text { les }\end{array}$ & $\begin{array}{l}\text { Conoce su confor- } \\
\text { mación y roles. }\end{array}$ & $\begin{array}{l}\text { La psicopedagoga y } \\
\text { la psicóloga tienen en } \\
\text { claro los roles de cada } \\
\text { una. }\end{array}$ & $\begin{array}{l}\text { Conocen su confor- } \\
\text { mación. Acceso a } \\
\text { los cargos: por con- } \\
\text { curso -acto público } \\
\text { docente, horas ti- } \\
\text { tulares, provisiona- } \\
\text { les o suplentes. Los } \\
\text { docentes dicen des- } \\
\text { conocer sus roles } \\
\text { porque "no vienen } \\
\text { mucho" al colegio. }\end{array}$ \\
\hline $\begin{array}{l}\text { Sobre funciones } \\
\text {-actividades y } \\
\text { tareas que lleva } \\
\text { a cabo el EOE } \\
\text { Grado de inter- } \\
\text { vención del EOE } \\
\text { en la institución }\end{array}$ & $\begin{array}{l}\text { A partir de la con- } \\
\text { vocatoria del Equi- } \\
\text { po directivo, se } \\
\text { deriva a la psicope- } \\
\text { dagoga por cues- } \\
\text { tiones académi- } \\
\text { cas y a la psicóloga } \\
\text { por temas de dis- } \\
\text { ciplina-conviven- } \\
\text { cia-vínculos. }\end{array}$ & $\begin{array}{l}\text { Prevención de situacio- } \\
\text { nes problemáticas indi- } \\
\text { viduales y grupales de } \\
\text { los alumnos, inclusión, } \\
\text { dificultades del apren- } \\
\text { dizaje y la socializa- } \\
\text { ción, alumnos con in- } \\
\text { tegración, estrategias } \\
\text { para profesores so- } \\
\text { bre el mejoramiento de } \\
\text { sus prácticas docentes, } \\
\text { Educación Sexual Inte- } \\
\text { gral (ESI), problemas } \\
\text { de convivencia. }\end{array}$ & $\begin{array}{l}\text { No todos conocen } \\
\text { las funciones y ac- } \\
\text { tividades del EOE. } \\
\text { Desconocen có- } \\
\text { mo se distribuyen } \\
\text { las intervenciones } \\
\text { y cuáles son las de } \\
\text { mayor conflictivi- } \\
\text { dad. }\end{array}$ \\
\hline
\end{tabular}




\begin{tabular}{|c|c|c|c|}
\hline $\begin{array}{l}\text { Percepción y } \\
\text { pensamientos } \\
\text { de los miembros } \\
\text { acerca del fun- } \\
\text { cionamiento del } \\
\text { EOE }\end{array}$ & $\begin{array}{l}\text { Coinciden en que el } \\
\text { trabajo que desa- } \\
\text { rrollan es muy bue- } \\
\text { no. Aunque desta- } \\
\text { can que deberían } \\
\text { estar más tiempo } \\
\text { en la institución. }\end{array}$ & $\begin{array}{l}\text { Mencionan como rasgo } \\
\text { destacable la coopera- } \\
\text { ción que existe con el } \\
\text { Equipo directivo. Re- } \\
\text { saltan, por otro lado, } \\
\text { escasez de personal y } \\
\text { de tiempo de encuen- } \\
\text { tro con profesores -di- } \\
\text { rectivos. }\end{array}$ & $\begin{array}{l}\text { Opiniones diver- } \\
\text { sas: para algunos, } \\
\text { es muy importan- } \\
\text { te el rol que cum- } \\
\text { plen, mientras que } \\
\text { otros no saben qué } \\
\text { responder pues no } \\
\text { los "ven nunca en } \\
\text { el colegio". }\end{array}$ \\
\hline
\end{tabular}

\section{Colegio B}

Tabla 4. Datos recolectados en el Colegio B

\begin{tabular}{|c|c|c|c|}
\hline Variables & Equipo directivo & EOE & Profesores \\
\hline $\begin{array}{l}\text { El grado de ar- } \\
\text { ticulación y vin- } \\
\text { culación con las } \\
\text { distintas estruc- } \\
\text { turas de la insti- } \\
\text { tución }\end{array}$ & $\begin{array}{l}\text { Mediante la inte- } \\
\text { racción permanen- } \\
\text { te con el EOE., los } \\
\text { docentes y las fa- } \\
\text { milias. Es parte in- } \\
\text { tegrante del Equipo } \\
\text { directivo. }\end{array}$ & $\begin{array}{l}\text { A través de la comu- } \\
\text { nicación institucional } \\
\text { (correo electrónico - } \\
\text { presencial), solicitudes } \\
\text { de intervención de los } \\
\text { profesores y familias y } \\
\text { reuniones con profeso- } \\
\text { res y directivos. }\end{array}$ & $\begin{array}{l}\text { Lo ubican en el } \\
\text { organigrama insti- } \\
\text { tucional conectado } \\
\text { horizontalmente } \\
\text { con el Equipo di- } \\
\text { rectivo. }\end{array}$ \\
\hline $\begin{array}{l}\text { Conformación } \\
\text { del EOE y sus } \\
\text { roles }\end{array}$ & $\begin{array}{l}\text { Psicólogo, psico- } \\
\text { pedagoga, y dos } \\
\text { orientadores que } \\
\text { son profesores del } \\
\text { colegio. }\end{array}$ & $\begin{array}{l}\text { Los orientadores son el } \\
\text { contacto directo con los } \\
\text { profesores y alumnos. } \\
\text { Derivación por caso: } \\
\text { psicopedagoga, temas } \\
\text { de aprendizaje; psicólo- } \\
\text { go, temas sociales-vin- } \\
\text { culares o disciplinarios. }\end{array}$ & $\begin{array}{l}\text { Conocen la confor- } \\
\text { mación y los roles. } \\
\text { La presencia de los } \\
\text { miembros del EOE } \\
\text { es visible para to- } \\
\text { dos los profesores. }\end{array}$ \\
\hline $\begin{array}{l}\text { Sobre funciones } \\
\text {-actividades y } \\
\text { tareas que Ileva } \\
\text { a cabo el EOE. } \\
\text { Grado de inter- } \\
\text { vención del EOE } \\
\text { en la institución }\end{array}$ & $\begin{array}{l}\text { Revisión del acuer- } \\
\text { do de convivencia } \\
\text { institucional, el } \\
\text { proyecto de edu- } \\
\text { cación sexual inte- } \\
\text { gral. Jornadas de } \\
\text { capacitación con } \\
\text { los docentes, reu- } \\
\text { niones con padres } \\
\text { y profesionales ex- } \\
\text { ternos. Inclusión } \\
\text { educativa. }\end{array}$ & $\begin{array}{l}\text { Seguimiento de las } \\
\text { trayectorias escolares, } \\
\text { proyectos de integra- } \\
\text { ción, vínculos entre } \\
\text { alumnos o grupos de } \\
\text { alumnos (disciplina), } \\
\text { orientación vocacional } \\
\text { (5to y 6to año), reu- } \\
\text { niones con las familias, } \\
\text { alumnos y profesores. } \\
\text { ESI, inclusión y admi- } \\
\text { sión de alumnos. }\end{array}$ & $\begin{array}{l}\text { Conocen las fun- } \\
\text { ciones, la distri- } \\
\text { bución de tareas y } \\
\text { roles que llevan a } \\
\text { cabo los miembros } \\
\text { del EOE. Recono- } \\
\text { cen la participa- } \\
\text { ción del EOE en la } \\
\text { revisión del acuer- } \\
\text { do institucional de } \\
\text { convivencia y el } \\
\text { proyecto educativo } \\
\text { institucional. }\end{array}$ \\
\hline
\end{tabular}




\begin{tabular}{|c|c|c|c|}
\hline $\begin{array}{l}\text { Percepción y } \\
\text { pensamientos } \\
\text { de los miembros } \\
\text { acerca del fun- } \\
\text { cionamiento del } \\
\text { EOE }\end{array}$ & $\begin{array}{l}\text { El trabajo que lle- } \\
\text { va adelante el EOE } \\
\text { es muy importan- } \\
\text { te para el colegio. } \\
\text { Es un apoyo para } \\
\text { nosotros, para los } \\
\text { docentes y las fa- } \\
\text { milias. }\end{array}$ & $\begin{array}{l}\text { Consideran que son un } \\
\text { pilar muy importante } \\
\text { que sostiene la relación } \\
\text { entre familia-colegio, } \\
\text { aunque comentan que } \\
\text { debería sumarse más } \\
\text { personal al equipo de } \\
\text { trabajo. }\end{array}$ & $\begin{array}{l}\text { El EOE es un so- } \\
\text { porte fundamental } \\
\text { para el docente. } \\
\text { Hay satisfacción } \\
\text { con la participa- } \\
\text { ción del EOE en las } \\
\text { situaciones donde } \\
\text { se demanda su in- } \\
\text { tervención. }\end{array}$ \\
\hline
\end{tabular}

\section{Análisis y discusión}

En el análisis y el detalle de las tareas que lleva adelante el EOE en el Colegio A, se reconoce que cumplen con los marcos prescriptivos-normativos establecidos por la provincia de Buenos Aires, mientras que, en la institución de gestión privada (Colegio $B$ ), el desempeño y funcionamiento del EOE, además de cumplir con los preceptos de la normativa oficial, se inscribe dentro de una organización escolar que aprende. Pues lo que resaltan en el Colegio B son las condiciones que postula Bolívar (2001) para que la escuela se conforme como una organización que aprende:

Si no hay proyectos de trabajo conjuntos, no hay base para el aprendizaje organizativo. Estos presuponen, una aceptación compartida de visiones y necesidades, que debe -entonces- ser el punto primero de actuación y, a su vez, ir provocando un cambio en la cultura escolar (por ejemplo, del individualismo). Justo entonces, se puede hablar de desarrollo de la organización, que cuando llega a institucionalizarse, por procesos internos y conjuntos de autorrevisión y planes de acción para el desarrollo, daría lugar a irse acercando a una organización que aprende. (p. 5)

Se comprende esta distinción con el Colegio A en la capacidad de poder comprender que el futuro es inestable, cambiante y no inmutable, predecible o estable. Aguerrondo (1996) menciona que esta estabilidad deviene en rigidez, imponiendo en la organización una férrea burocracia. Por lo tanto, se "requiere ahora una reconversión. Esto quiere decir que se necesita encontrar otra fuente de estabilidad que permita enfrentar los cambios de manera más flexible" (p. 5).

Se presenta, también, una relación entre intervención y margen de autonomía que reviste características distintas en cada colegio. En el Colegio A, la intervención del EOE está bien definida y el margen de autonomía está acotado. En las instituciones educativas estatales, "además de la escasez de recursos financieros, poseen una creciente burocratización de la tarea educadora y en cierta forma una limitación (paradójicamente estatal) para aplicar nuevas herramientas administrativas y pedagógicas" (Narodowski y Andrada, 2004, p. 212). Prevalece, en esta institución, un modelo de gestión normativo que establece un estilo verticalista y "en la cual, por una parte, se encuentran los sujetos encargados de planificar $y$, por otra parte, se encuentran los sujetos encargados de administrar o ejecutar los planes" (Casassus, 2002, p. 52). Este tipo de gestión manifiesta una comprensión de tipo lineal del fututo, o sea, desde lo que se planifica y hacia la concreción de los objetivos, se recorre un camino que no 
sufre ningún tipo de obstáculos. El mismo autor, al referirse a los aspectos teóricos y técnicos de este modelo, comenta lo siguiente:

Desde el punto de vista teórico, en esta perspectiva el futuro es único y cierto. Desde el punto de vista técnico la planificación consistió en la aplicación de técnicas de proyección del presente hacia el futuro. Este modelo es la expresión de un modelo racionalista weberiano, con un alto nivel de abstracción y donde la dinámica propia de la sociedad estaba ausente. (Casassus, 2002, p. 54)

En la escuela $B$, también se evidencia claridad en el campo de intervención, pero con un margen de autonomía amplio. La explicación de ello la aportan Narodowski y Andrada (2004) al explicitar que las instituciones educativas privadas constituyen subsistemas que se presentan indeterminadamente autónomos con la burocracia, la administración y el control estatal, lo que genera que, en estos subsistemas, existan grandes márgenes de autonomía para establecer definiciones respecto a las actividades educativas, como los horarios de las clases, la forma de contratación, la elección del personal, docentes, etc. Esta cuestión es importante, pues se deben establecer claramente los roles y funciones del EOE, los límites de actuación profesional, consensos y acuerdos con el resto de los actores institucionales para evitar conflictos en relación con la superposición de roles y funciones. Por lo tanto, la gestión se enmarca en el modelo estratégico situacional. La estrategia se entiende como las acciones que se realizan para conseguir un determinado fin y la situación es donde interactúan el actor educativo y la acción que este lleva a cabo. Por lo tanto, se generarán muchas situaciones en la realidad que van a depender de cómo esté situado el actor educativo y la situación en la que esté (Matus, 1987). En síntesis, este modelo "se preocupa del análisis y del abordaje de los problemas en el trayecto hacia el objetivo o el futuro deseado, la gestión se presenta como un proceso de resolución de nudos críticos de problemas" (Casassus, 2002, p. 56).

Con respecto a la dinámica del funcionamiento del EOE y la articulación con el equipo directivo en cada institución, se reconoce que, en el Colegio $A$, está determinado por la derivación. Cuando interviene el $E O E$, lo hace atendiendo al alumno "problema" que presenta alguna dificultad, lo que evidencia una intervención de tipo clínica-médica. El equipo directivo, los profesores y el EOE colocan las dificultades y toda la responsabilidad en los alumnos. Si bien esto no se verbaliza, se constata en la modalidad que adoptan los diversos abordajes y prácticas que lleva a cabo el EOE. Como consecuencia de ello, las funciones que se desarrollan están definidas por la demanda de atención de casos individuales de alumnos que presentan problemáticas en los aprendizajes o en la conducta, solicitada por la Dirección.

Se aprecia un desalojo de la dimensión pedagógica, ya que el alumno "problemático" manifiesta toda una serie de conductas cuya explicación se centra en los aspectos personales-familiares y sociales y no se consideran en el análisis las variables pedagógico-didácticas ni las prácticas institucionales. No entra en consideración una revisión crítica del proceso de enseñanza-aprendizaje. Las prácticas escolares de la institución, su tradición y costumbres posibilitan que resulte más simple la demanda-derivación al EOE en vez de reflexionar, investigar y profundizar sobre nuevas estrategias didácticas y metodológicas a implementar. En síntesis, como lo refiere Baquero (2002), la explicación de ello es porque no se manifiestan, en esta institución, atisbos del giro contextualista o situacional, lo que genera que las intervenciones del EOE sean con- 
ceptualizadas desde "una propiedad más de las situaciones que de los sujetos a título sólo individual" (p. 65).

Si en el Colegio A la modalidad de trabajo del EOE se da dentro de una estructura rígida y de organización vertical cuyo denominador común en sus intervenciones es la derivación, en el Colegio B, los profesores, el EOE y el equipo de conducción definen y encuadran las dificultades que presentan los alumnos como problemáticas individuales-grupales e institucionales. El colegio favorece que los actores institucionales compartan intereses, busquen y propongan alternativas para la solución de las diversas problemáticas que se presenten. Construyen, de manera colaborativa, conocimiento, que se va enriqueciendo con los distintos puntos de vista de los profesores, de los miembros del EOE y del equipo directivo. Es una organización "donde la gente expande continuamente su aptitud para crear los resultados que desea, donde se cultivan nuevos y expansivos patrones de pensamiento, donde la aspiración colectiva queda en libertad, y donde la gente continuamente aprende a aprender en conjunto" (Senge, 1990 , p. 3).

Finalmente, vale aclarar lo que remarca Hargreaves (1998) cuando expresa que "trabajar conjuntamente no es simplemente una forma de construir relaciones y buscar una resolución colectiva. También es una fuente de aprendizaje" (p. 27). De esta manera, se establece, en el Colegio $B$, un cambio que va desde la acción aislada, de dependencia y la falta de compromiso profesional hacia la colaboración entre los profesionales, que tendrán autonomía, fomentarán la participación colectiva y la responsabilidad profesional (Romero, 2008), lo que finalmente transforma a esta institución en una comunidad de aprendizaje.

\section{CONCLUSIONES}

Este trabajo permite vislumbrar qué ocurre con los Equipos de Orientación Escolar en dos escuelas secundarias de la provincia de Buenos Aires. A su vez, es un punto de partida sobre el cual se puede, por un lado, comprender cuáles son las adversidades y los aspectos que favorecen su óptimo funcionamiento en la institución educativa y, por otro lado, es un punto de referencia para otros estudios que puedan profundizar y ampliar el horizonte del EOE. Agrega, también, un análisis que los estudios llevados a cabo en nuestro país por Bayeto (2016), Greco (2014 y 2015), Bertoglio, Corizzo y Steiman (2017) y los trabajos de Sallán (1985) y Vélaz de Medrano et al. (2013), producidos en España, no exhiben: el contraste y comparación entre colegios públicos y privados de los Equipos de Orientación Escolar.

En el colegio de gestión estatal, lo investigado en este estudio coincide, por un lado, con las problemáticas del EOE descritas por Bayeto (2016). Así, se pudo verificar la burocratización, el automatismo de las derivaciones y la psicopatologización de los problemas educativos. Y, por otro lado, con Greco (2014), sobre la tendencia del EOE en ofrecer respuestas individuales a situaciones que no dejan de ser institucionales. En el colegio A, se evidenció que los miembros del EOE desarrollan las actividades de manera aislada, no están integrados a espacios institucionales; por lo tanto, existe un desconocimiento para el resto de los miembros de la organización y una falta de información de cómo funciona el EOE. Es un contexto predominantemente tecnocrático, donde las funciones deben ser ejecutadas por expertos y tienen que ajustarse a lo 
normativo-prescriptivo, que abona al trabajo individual y la privacidad y donde no hay trabajo en equipo, sino fragmentación.

Por su parte, en el colegio de gestión privada, se observa una coincidencia con lo señalado por Greco (2015) cuando señala que la reformulación en los EOE de los colegios privados, de sus prácticas y concepciones es lo que desembocó en modelos de intervención distintos para poder dar respuesta a la problemática presentada en el ámbito de las instituciones educativas estatales. El EOE del Colegio B da una imagen de articulación con el equipo directivo y los demás actores institucionales saben cómo funciona y las tareas que llevan a cabo. De esta manera, esta institución promueve prácticas colaborativas que establecen el trabajo en equipo. De esta manera, se evita la tendencia a que el EOE esté aislado, ya que el trabajo así establecido favorece el intercambio profesional, brinda apoyo y acompañamiento mutuo entre los actores institucionales y estimula la solidaridad, la cooperación, la autonomía y el aprendizaje colectivo. En cierta manera, aunque en este aspecto se puede ahondar más en posteriores investigaciones, lo anterior está en sintonía con lo expuesto por Bertoglio, Corizzo y Steiman (2017) cuando argumentan que la evolución del término gabinete a lo que hoy conocemos como Equipo de Orientación Escolar conlleva el pasaje de prácticas centradas en el alumno-problema al tratamiento de las problemáticas de modo interdisciplinario y en equipo, entendiendo al alumno en contexto y en situación pedagógica. Y cuando se permite la colaboración, la participación y el trabajo en conjunto, que genera una reflexión sobre las propias prácticas, se pueden establecer estrategias metodológico-didácticas más adecuadas para las problemáticas que deben ser atendidas por parte del EOE. Esto permite expresar que lo observado en el Colegio B está en sintonía con los postulados de una organización que aprende.

En el Colegio $A$, si bien hay algunos actores institucionales que manifestaron ver poco o nada a los miembros del EOE, la mayoría expresó que la función que cumple es muy importante. La realidad es distinta en el colegio privado, donde todos los actores coinciden en la importancia de su función y resaltan que es un apoyo fundamental para la tarea cotidiana en la escuela. Esto último está en concordancia con Sallán (1985), ya que, en el Colegio B, la función del EOE se piensa desde una acción en equipo, donde se promueve la coordinación del conjunto de actores educativos. Cabe destacar una coincidencia en ambos colegios acerca de las cuestiones que atentan sobre el óptimo funcionamiento del EOE. La falta de disponibilidad de tiempo y más recursos humanos para conformar el EOE son los dos aspectos más resaltados, aunque es pertinente analizar dos aspectos. Por un lado, en el Colegio A, no se mencionó como impedimento para el ideal funcionamiento del EOE la falta de participación en las decisiones y en los consensos institucionales. Y, por otro lado, en el Colegio B, quedaría pendiente analizar en trabajos nuevos lo que menciona Sallán (1985) con respecto a que, además de la falta de personal y la disponibilidad de tiempo, el sentido individual y las diferencias ideológicas de los actores serían otros dos factores que pueden impedir el óptimo funcionamiento del EOE.

Las cuestiones mencionadas anteriormente permiten apreciar un mejor funcionamiento del EOE en el colegio de gestión privada que en el público, pues la autonomía y el margen de flexibilidad que exhibe en sus prácticas, el estilo de gestión colaborativo del equipo directivo, el rol de los orientadores y su participación como miembros del EOE y la diferencia de matrícula escolar a la que se debe atender -trescientos alumnos contra casi mil trescientos en el colegio A- son las características que lo fundamentan. Finalmente, para futuros trabajos sobre el tema, quedan abiertas algunas preguntas 
para profundizar: ¿En qué medida los hallazgos de este estudio pueden ser comunes a otras escuelas secundarias de la provincia de Buenos Aires? ¿Se mantienen las diferencias encontradas en el funcionamiento de los dos EOE estudiados al analizar otros colegios de gestión estatal y privada? ¿Qué pasa en colegios de otras jurisdicciones y de otras provincias?

\section{Referencias bibliográficas}

Aguerrondo, I. (Ed.). (1996). La escuela como organización inteligente. Buenos Aires: Troquel.

Baquero, R. (2000). Lo habitual del fracaso o el fracaso de lo habitual. Rosario: Homo Sapiens.

Baquero, R. (2002). Del experimento escolar a la experiencia educativa. La transmisión educativa desde una perspectiva psicológica situacional. Perfiles educativos. 24(97-98), 57-75.

Bayeto, G. (2016). Los Equipos de Apoyo y Orientación Escolar y sus normativas desde la perspectiva de las políticas públicas de inclusión educativa: El caso de los Departamentos de Orientación Educativa (DOE) en escuelas medias públicas de la Ciudad de Buenos Aires. Problemas y debates para la investigación sobre sus prácticas. Investigaciones en Psicología, 21(1), 13-25. Recuperado de: http://www.psi.uba.ar/investigaciones/revistas/investigaciones/indice/trabajos_ completos/anio21_1/bayeto.pdf.

Bello, C. (2010). ABC Orientadores Sociales. Equipos de Orientación Escolar. Revista de la Dirección General de Cultura y Educación de la Provincia de Buenos Aires, 3(7), 37-39. Recuperado de: http://servicios.abc.gov.ar/lainstitucion/revistacomponents/revista/archivos/abc-delaeducacion/ numero07/ArchivosParaDescargar/ABCOrientadoresSociales.pdf

Bertoglio, M., Corizzo, M. \& Steiman, B. (2017). Los Equipos de Orientación Escolar en el Sistema Educativo: La construcción de legajos grupales: Una mirada Interdisciplinaria. Ingenium, 3(6), $18-20$.

Bolívar, A. (2001). Los Centros Educativos como organizaciones que aprenden: Una mirada crítica. Contexto Educativo, 3(18), 1-11.

Casassus, J. (2002). Problemas de la Gestión Educativa en América Latina: la tensión entre los paradigmas de tipo A y el tipo B. Em Aberto, 19(75), 49-69.

Castorina, J. (2005). Las prácticas sociales en la formación del sentido común. La naturalización en la psicología. En S. Llomovatte y C. Kaplan (comps.), Desigualdad educativa. La naturaleza como pretexto (pp. 21-39). Buenos Aires: Novedades Educativas.

Cervini, R. (2002). Desigualdades en el logro académico y reproducción cultural en Argentina. Revista Mexicana de Investigación Educativa, 7(16), 445-500.

Dirección General de Cultura y Educación, Buenos Aires (2007). Ley de Educación Provincial No 13.688. Recuperado de: http://servicios.abc.gov.ar/docentes/capacitaciondocente/pruebadeseleccion2008/presentacion/Normativa\%20Comun/Ley\%2013688-07.pdf.

Dirección General de Cultura y Educación, Buenos Aires (2007). Ley 13688 de Educación de la Provincia de Buenos Aires. La Plata: Dirección General de Cultura y Educación. Recuperado de: http://servicios.abc.gov.ar/docentes/capacitaciondocente/pruebadeseleccion2008/presentacion/ Normativa\%20Comun/Ley\%2013688-07.pdf. 
Dirección General de Cultura y Educación, Buenos Aires (2008). Disposición 76/08. Recuperado de: http://www.abc.gov.ar/psicologia/sites/default/files/documentos/disposicion_ndeg_76-08. rol_del_equipo_de_orientacion_escolar.pdf.

Dirección General de Cultura y Educación, Buenos Aires (2009). Disposición 01/09. Recuperado de: http://www.servicios.gov.ar/lainstitucion/sistemaeducativo/psicologiasse/normativa/documentosdescarga/disp 0109 equipos de distrito.pdf.

Dirección General de Cultura y Educación, Buenos Aires (2012). Reglamento General de la Instituciones Educativas de la provincia de Buenos Aires. Recuperado de: http://servicios2.abc.gov. ar/lainstitucion/organismos/consejogeneral/reglamento_general/reglamento_general.pdf.

Fernández, M. B. (2002). Crisis, instituciones educativas y transformaciones culturales. Revista Tram(p)as de la Comunicación y la Cultura, 1(5), 16-19.

Greco, M. (2014). Intervenciones de los Equipos de Orientación Escolar: Entre la habilitación de los sujetos y la creación condiciones institucionales. VI Congreso Internacional de Investigación y Práctica Profesional en Psicología XXI Jornadas de Investigación Décimo Encuentro de Investigadores en Psicología del MERCOSUR. Facultad de Psicología - Universidad de Buenos Aires, Buenos Aires. Recuperado de: http://baseries.flacso.org.ar/uploads/productos/1260_01.pdf.

Greco, M. (2015). Las trayectorias educativas desde la perspectiva de una psicología educacional contemporánea. VII Congreso Internacional de Investigación y Práctica Profesional en Psicología XXII Jornadas de Investigación XI Encuentro de Investigadores en Psicología del MERCOSUR. Facultad de Psicología - Universidad de Buenos Aires, Buenos Aires. Recuperado de: https://www. aacademica.org/000-015/450.pdf.

Hargreaves, A. (1998). Paradojas del cambio. La renovación de la escuela posmoderna. Sevilla: Kikiriki Cooperativa Educativa.

Matus, C. (1987). Política, planificación y gobierno. Washington D.C.: Naciones Unidas. Programa de Desarrollo.

Maykut, P. \& Morehouse, R. (1994). Beginning qualitative research a philosophical and practical guide. Washington D.C.: Falmer Press.

Ministerio de Educación de la Ciudad Autónoma de Buenos Aires, Argentina (2009). Documento sobre Prácticas de Inclusión. Ciudad Autónoma de Buenos Aires: Autor. Narodowski, M. \& Andrada, M. (2004). Monopolio Estatal y Descentralización Educativa. Una Exploración en América Latina. Revista de Educación, 333, 197-219. Recuperado de: http://www.educacionyfp.gob.es/ dam/jcr:365d350f-3f16-4b3d-8cd2-992524e9d475/re33309-pdf.pdf.

Ministerio de Cultura y Educación Nacional, Argentina (2006). Ley de Educación Nacional 26.206. Recuperado de: http://www.secgral.unsl.edu.ar/wp-content/uploads/docs/Ley-26206-de-Educacion-Nacional.pdf.

Pezzenati, D. (2019). Los Equipos de Orientación Escolar en el nivel medio: Análisis de su funcionamiento en una escuela secundaria de gestión pública y otra de gestión privada de la provincia de Buenos Aires. Tesis de Maestría, Universidad Di Tella, Buenos Aires. Texto completo: https:// docs.google.com/document/d/1bj5cn11Dv3kMLY56XdEp0tpYUuD_vPMdKy131413x4E/edit?us$\mathrm{p}=$ sharing.

Romero, C. (2008). Hacer de una escuela, una buena escuela. Buenos Aires: Aique.

Ruiz del Cerro, J. (2000). La entrevista en profundidad en la investigación cualitativa. Presentación gráfica de sus textos. XII Congreso Nacional y I Iberoamericano de Pedagogía. Tomo II (pp. 71-74). Madrid: Sociedad Española de Pedagogía. 
Sallán, J. (1985). El Departamento de Orientación desde la perspectiva de la Organización Escolar. Educar, 8, 33-60. Recuperado de: https://ddd.uab.cat/pub/educar/0211819Xn8/0211819Xn8p33. pdf.

Senge, P. (1990). La quinta disciplina. Cómo impulsar el aprendizaje en la organización inteligente. Buenos Aires: Granica/Vergara.

Sileoni, A. (2014). Los equipos de orientación en el sistema educativo. La dimensión institucional de la intervención. La Dimensión Institucional de la Intervención. Ciudad Autónoma de Buenos Aires: Ministerio de Educación de la Nación. Recuperado de: https://cdn.educ.ar/repositorio/ Download/file?file_id=3d823c14-8d1d-48ab-bf81-b58fee28b2cc.

Vélaz de Medrano Ureta, C., Manzanares Moya, A., López Martín, E. \& Manzano Soto, N. (2013). Competencias y formación de los Orientadores Escolares: Estudio Empírico en nueve Comunidades Autónomas. Revista de Educación, 1, 261-292. Recuperado de: http://repositorio.minedu.gob.pe/bitstream/handle/123456789/2416/2013_V\%C3\%89LAZDEMEDRANO_Competencias $\% 20$ y $\% 20$ formaci\%C3\%B3n\%20de $\% 20$ los $\% 20$ orientadores $\% 20$ escolares $\% 20$ estudio $\% 20$ emp $\%$ C3\%ADrico $\% 20$ en $\% 20$ nueve $\% 20$ comunidades $\% 20$ aut $\%$ C3\%B3nomas.pdf?sequen$\mathrm{ce}=1$ \&isAllowed $=$.

Vicente, M. (2016). Estructuras organizacionales de la escuela secundaria. IX Jornadas de Sociología de la Universidad Nacional de La Plata. Universidad Nacional de La Plata, Ensenada, Argentina. Recuperado de: http://www.memoria.fahce.unlp.edu.ar/trab_eventos/ev.9035/ev.9035.pdf. 